\title{
An Optimal Consumption and Investment Problem with Quadratic Utility and Subsistence Consumption Constraints: A Dynamic Programming Approach
}

\section{Yong Hyun Shin ${ }^{a}$, Jung Lim Koo ${ }^{b}$ and Kum-Hwan Roh ${ }^{c}$}

${ }^{a}$ Department of Mathematics 8 Research Institute of Natural Sciences, Sookmyung Women's University

04310 Seoul, Republic of Korea

${ }^{b}$ School of Law, University of Texas at Austin

Austin, 78705 Texas, USA

${ }^{c}$ Department of Mathematics, Hannam University

34430 Daejeon, Republic of Korea

E-mail(corresp.): khroh@hnu.kr

E-mail: yhshin@sookmyung.ac.kr

E-mail: koolimy@gmail.com

Received October 16, 2017; revised September 7, 2018; accepted September 12, 2018

\begin{abstract}
In this paper, we analyze the optimal consumption and investment problem of an agent who has a quadratic-type utility function and faces a subsistence consumption constraint. We use the dynamic programming method to solve the optimization problem in continuous-time. We further provide the sufficient conditions for the optimization problem to be well-defined.
\end{abstract}

Keywords: portfolio selection, quadratic utility, subsistence consumption constraints, dynamic programming method.

AMS Subject Classification: 91G10; 49L20; 60H30.

\section{Introduction}

We consider a continuous-time optimal consumption and portfolio selection problem of an agent who has a quadratic utility function and faces a subsistence consumption constraint. We solve the problem using the dynamic programming

Copyright (c) 2018 The Author(s). Published by VGTU Press

This is an Open Access article distributed under the terms of the Creative Commons Attribution License (http://creativecommons.org/licenses/by/4.0/), which permits unrestricted use, distribution, and reproduction in any medium, provided the original author and source are credited. 
method and find closed-form solutions for the value function and the optimal consumption and investment policies.

Our paper follows the literature that studies optimal consumption and portfolio selection in continuous time using the dynamic programming method. Merton [13] solved a continuous-time optimal consumption and portfolio selection problem for an agent with constant relative risk aversion (CRRA) utility using the dynamic programming method. Merton [14] expanded [13] to consider the case of an agent with hyperbolic absolute risk aversion (HARA) utility. Quadratic utility is a member of the HARA class of utilities introduced in Merton [14]. Karatzas et al. [7] further expanded the literature by using dynamic programming to solve an optimal consumption and portfolio selection problem for an agent with general utility.

Quadratic utility is widely used in mean-variance analysis. Markowitz [12] insisted that quadratic utility provides a good approximation to other utility functions in portfolio choice models. Hanoch and Levy [5] and Buccola [1] compare the risk aversion of quadratic utility with cubic and exponential utility respectively. Hanoch and Levy [5] provide an optimal efficiency criteria for a portfolio selection problem when the agent's utility is quadratic. Buccola [1] finds that the choice between quadratic and exponential utility have no effect on optimal portfolio selection if the corresponding absolute risk aversion coefficients at the optimal solution are equal.

Our paper also follows the literature studying subsistence consumption constraints. A subsistence consumption constraint provides a lower bound for which a decline in consumption below this level is not tolerated. As Dybvig [3] notes, such a constraint can be interpreted as an exterme form of habit formation. The existence of a subsistence consumption constraint has a significant impact on the agent's optimal consumption and investment policies, especially when the constraints are binding. Cox and Huang [2], Gong and Li [4], Huang and Pagès [6], Koo et al. [8], Lakner and Nygren [9], Lee and Shin [10], Lim et al. [11], Shim and Shin [15], Shin and Lim [16], Shin et al. [17], and Yuan and $\mathrm{Hu}[18]$ have studied optimal consumption and portfolio selection problems with subsistence consumption constraints.

Koo et al. [8] also solved an optimal consumption and portfolio selection problem for an agent with quadratic utility and subsistence consumption constraints. However, Koo et al. [8] solved the problem using the martingale method, while we use the dynamic programming method. More significantly, we can replicate the results of Koo et al. [8] without the assumption $\rho-2 r+\theta^{2}>0$. We further provide a verification theorem that shows that the value function obtained using the dynamic programming method is the same as the value function obtained using the martingale method in Koo et al. [8].

The rest of this paper proceeds as follows. In Section 2, we describe the financial market. We use the dynamic programming method to solve our optimization model in Section 3. Section 4 provides some properties of the optimal solutions and Section 5 concludes. 


\section{The financial market}

We assume that there are two assets in the financial market: A riskless asset with constant interest rate $r>0$ and a risky asset $S_{t}$ governed by the following geometric Brownian motion:

$$
d S_{t}=\mu S_{t} d t+\sigma S_{t} d B_{t}
$$

where $\mu>0$ and $\sigma>0$ are constants and $B_{t}$ is a standard Brownian motion defined on a complete probability space $(\Omega, \mathcal{F}, \mathbb{P})$ and $\left\{\mathcal{F}_{t}\right\}_{t>0}$ is the $\mathbb{P}$ augmentation of the filtration generated by the standard Brownian motion $\left\{B_{t}\right\}_{t \geq 0}$.

Let $\pi_{t}$ be the amount invested in the risky asset at time $t$ and $c_{t}$ be consumption at time $t$. A portfolio process $\left\{\pi_{t}\right\}_{t>0}$ is a measurable and adapted process with respect to $\left\{\mathcal{F}_{t}\right\}_{t \geq 0}$, and a consumption process $\left\{c_{t}\right\}_{t \geq 0}$ is a measurable and adapted positive process with respect to $\left\{\mathcal{F}_{t}\right\}_{t \geq 0}$. They satisfy the following mathematical conditions:

$$
\int_{0}^{t} \pi_{s}^{2} d s<\infty \text { and } \int_{0}^{t} c_{s} d s<\infty, \text { for all } t \geq 0 \text { a.s. }
$$

Let $X_{t}$ be the agent's wealth process at time $t$. It evolves according to the following stochastic differential equation (SDE):

$$
d X_{t}=\left[r X_{t}+\pi_{t}(\mu-r)-c_{t}\right] d t+\sigma \pi_{t} d B_{t}
$$

with initial endowment $X_{0}=x$. Furthermore we assume that there is a subsistence consumption constraint which restricts the minimum consumption level such that

$$
c_{t} \geq \Gamma, \text { for all } t \geq 0,
$$

where $\Gamma>0$ is a fixed constant lower bound of consumption. From this constraint (2.3), we have the following condition for the initial endowment: $x>\Gamma / r$.

\section{The optimization problem with a dynamic programming approach}

Now we consider the following optimization problem. The infinite-lived agent wants to maximize her expected discounted lifetime utility:

$$
V(x):=\sup _{(c, \pi) \in \mathcal{A}(x)} \mathbb{E}\left[\int_{0}^{\infty} e^{-\rho t} u\left(c_{t}\right) d t\right]
$$

subject to the wealth constraint (2.2) and the subsistence consumption constraint (2.3). $\mathcal{A}(x)$ is the class of all admissible controls

$$
(c, \pi):=\left(\left(c_{t}\right)_{t \geq 0},\left(\pi_{t}\right)_{t \geq 0}\right)
$$

at initial wealth $x$ with $x>\Gamma / r$ and each control $(c, \pi)$ is subject to the constraints $(2.1)$ and (2.3). Here, $\rho>0$ is a subjective discount factor and $u(\cdot)$ 
is a quadratic-type utility function defined by $u(c):=c-R c^{2}$, where $R>0$ is a constant. We also assume that the wealth process $X_{t}$ at time $t$ should satisfy the following transversality condition:

$$
\lim _{t \rightarrow \infty} e^{-\rho t} V\left(X_{t}\right)=0 .
$$

By the dynamic programming principle, the value function $V(x)$ in $(3.1)$ satisfies the following Bellman equation

$$
\rho V(x)=\max _{c \geq \Gamma, \pi}\left[(r x+\pi(\mu-r)-c) V^{\prime}(x)+\frac{1}{2} \sigma^{2} \pi^{2} V^{\prime \prime}(x)+c-R c^{2}\right] .
$$

From the first order conditions (FOCs) of the Bellman equation (3.3), we derive the candidate optimal consumption and portfolio policies $\left(c^{*}, \pi^{*}\right)$ as follows:

$$
c^{*}= \begin{cases}\Gamma, & \text { if } \Gamma / r<x<\widetilde{x} \\ \left(1-V^{\prime}(x)\right) /(2 R), & \text { if } \widetilde{x} \leq x<\bar{x} \quad, \quad \pi^{*}=-\frac{\theta}{\sigma} \frac{V^{\prime}(x)}{V^{\prime \prime}(x)}, \\ \bar{c}=1 /(2 R), & \text { if } x \geq \bar{x}\end{cases}
$$

where $\theta:=(\mu-r) / \sigma$ is the market price of risk, $\widetilde{x}$ is the threshold wealth level which corresponds to a subsistence consumption level $\Gamma$, and $\bar{x}=1 /(2 r R)$ is the wealth level that can support the bliss level of consumption $\bar{c}$ (for the concrete forms of the boundary wealth levels, refer to Koo et al. [8]).

Remark 1. For later use, we define two quadratic equations:

$$
f(m):=r m^{2}-\left(\rho+r+\theta^{2} / 2\right) m+\rho=0,
$$

with two roots $m_{1}$ and $m_{2}$ satisfying $m_{1}>1>m_{2}>0$ and

$$
g(n):=\theta^{2} n^{2} / 2+\left(\rho-r+\theta^{2} / 2\right) n-r=0,
$$

with two roots $n_{1}$ and $n_{2}$ satisfying $n_{1}>0$ and $n_{2}<-1$. The roots of the two quadratic equations (3.5) and (3.6) have the following relationship:

$$
n_{2}=\frac{1}{m_{2}-1} \text { and } m_{2}=\frac{n_{2}+1}{n_{2}} .
$$

Theorem 1. The value function $V(\cdot)$ of the optimization problem (3.1) is given by

$$
V(x)=\left\{\begin{array}{l}
C_{2}(x-\Gamma / r)^{m_{2}}+\left(\Gamma-R \Gamma^{2}\right) / \rho, \text { if } \Gamma / r<x<\widetilde{x}, \\
\frac{r-\theta^{2} n_{1} / 2}{\rho} D_{1}(1-2 R \xi)^{n_{1}+1}+\frac{(1-2 R \xi)^{2}}{4 R\left(\rho-2 r+\theta^{2}\right)}+\frac{1}{4 \rho R}, \quad \text { if } \widetilde{x} \leq x<\bar{x}, \\
1 /(4 \rho R), \text { if } x \geq \bar{x},
\end{array}\right.
$$

where

$$
\begin{aligned}
D_{1} & =\frac{\rho-r m_{2}+\theta^{2}}{2 r R\left(\rho-2 r+\theta^{2}\right)\left(n_{1}\left(m_{2}-1\right)-1\right)}(1-2 R \Gamma)^{1-n_{1}}, \\
\widetilde{x} & =D_{1}(1-2 R \Gamma)^{n_{1}}-\frac{1}{\rho-2 r+\theta^{2}} \Gamma+\frac{\rho-r+\theta^{2}}{2 r R\left(\rho-2 r+\theta^{2}\right)} \\
& =n_{1}\left(m_{2}-1\right) D_{1}(1-2 R \Gamma)^{n_{1}}+\frac{\left(m_{2}-1\right)(1-2 R \Gamma)}{2 R\left(\rho-2 r+\theta^{2}\right)}+\frac{\Gamma}{r},
\end{aligned}
$$




$$
C_{2}=\frac{1-2 R \Gamma}{m_{2}}\left(\widetilde{x}-\frac{\Gamma}{r}\right)^{1-m_{2}}>0,
$$

and $\xi$ is determined from the algebraic equation

$$
x=D_{1}(1-2 R \xi)^{n_{1}}-\frac{1}{\rho-2 r+\theta^{2}} \xi+\frac{\rho-r+\theta^{2}}{2 r R\left(\rho-2 r+\theta^{2}\right)} .
$$

Proof. For $\Gamma / r<x<\widetilde{x}$, substituting the FOCs (3.4) into equation (3.3) implies

$$
\rho V(x)=(r x-\Gamma) V^{\prime}(x)-\frac{1}{2} \theta^{2} \frac{\left(V^{\prime}(x)\right)^{2}}{V^{\prime \prime}(x)}+\Gamma-R \Gamma^{2} .
$$

From equation (3.8), we obtain the solution $V(x)$ as follows:

$$
V(x)=C_{2}\left(x-\frac{\Gamma}{r}\right)^{m_{2}}+\frac{\Gamma-R \Gamma^{2}}{\rho}, \text { for } \Gamma / r<x<\widetilde{x},
$$

where $C_{2}$ is a constant, and $m_{1}$ and $m_{2}$ satisfying $m_{1}>1>m_{2}>0$ are two roots of the quadratic equation (3.5). We will show that $C_{2}>0$ later (see $(3.19))$.

For $x \geq \bar{x}$, optimal consumption is constant at the bliss level $\bar{c}$, and the agent has zero investment in the risky asset. Thus the value function $V(x)$ is obtained from the Bellman equation (3.3) as follows:

$$
V(x)=1 /(4 \rho R), \quad \text { for } \quad x \geq \bar{x} .
$$

For $\widetilde{x} \leq x<\bar{x}$, we assume that optimal consumption $c=C(x)$ is a function of wealth and $X(\cdot)=C^{-1}(\cdot)$, that is, $X(c)=X(C(x))=x$. Then, from the FOCs (3.4), we have

$$
V^{\prime}(x)=1-2 R C(x), \quad V^{\prime \prime}(x)=-2 R / X^{\prime}(c) .
$$

Plugging the FOCs (3.4) and the equations (3.10) into equation (3.3) implies

$$
\rho V(X(c))=r(1-2 R c) X(c)+\frac{\theta^{2}(1-2 R c)^{2}}{4 R} X^{\prime}(c)+R c^{2} .
$$

Differentiating equation (3.11) with respect to $c$, we obtain the following equation

$$
\frac{\theta^{2}(1-2 R c)^{2}}{4 R} X^{\prime \prime}(c)-\left(\rho-r+\theta^{2}\right)(1-2 R c) X^{\prime}(c)-2 r R X(c)+2 R c=0 .
$$

Thus we obtain the solution to the second order ordinary differential equation $(3.12)$

$$
X(c)=D_{1}(1-2 R c)^{n_{1}}-\frac{1}{\rho-2 r+\theta^{2}} c+\frac{\rho-r+\theta^{2}}{2 r R\left(\rho-2 r+\theta^{2}\right)},
$$

where $D_{1}$ is a constant and $n_{1}>0$ is one of two roots of the quadratic equation (3.6). Substituting $X(c)$ in (3.13) into (3.11) implies

$V(x)=\frac{r-\frac{1}{2} \theta^{2} n_{1}}{\rho} D_{1}(1-2 R c)^{n_{1}+1}+\frac{(1-2 R c)^{2}}{4 R\left(\rho-2 r+\theta^{2}\right)}+\frac{1}{4 \rho R}$, for $\tilde{x} \leq x<\bar{x}$. 
Substituting $c=\Gamma$ into the function $X(\cdot)$ and the derivative of $X(\cdot)$ in (3.13) implies

$$
\begin{aligned}
& \widetilde{x}=X(\Gamma)=D_{1}(1-2 R \Gamma)^{n_{1}}-\frac{1}{\rho-2 r+\theta^{2}} \Gamma+\frac{\rho-r+\theta^{2}}{2 r R\left(\rho-2 r+\theta^{2}\right)}, \\
& X^{\prime}(\Gamma)=-2 n_{1} R D_{1}(1-2 R \Gamma)^{n_{1}-1}-1 /\left(\rho-2 r+\theta^{2}\right) .
\end{aligned}
$$

From (3.9) and (3.10), $C^{1}$ - and $C^{2}$ - conditions of $V(x)$ at $\widetilde{x}$ imply the following equations:

$$
\begin{aligned}
& V^{\prime}(\widetilde{x})=m_{2} C_{2}(\widetilde{x}-\Gamma / r)^{m_{2}-1}=1-2 R \Gamma, \\
& V^{\prime \prime}(\widetilde{x})=m_{2}\left(m_{2}-1\right) C_{2}(\widetilde{x}-\Gamma / r)^{m_{2}-2}=-2 R / X^{\prime}(\Gamma) .
\end{aligned}
$$

From (3.15) and (3.16), we obtain

$$
\widetilde{x}=n_{1}\left(m_{2}-1\right) D_{1}(1-2 R \Gamma)^{n_{1}}+\frac{\left(m_{2}-1\right)(1-2 R \Gamma)}{2 R\left(\rho-2 r+\theta^{2}\right)}+\frac{\Gamma}{r} .
$$

From (3.14) and (3.17), we also derive

$$
D_{1}=\frac{\rho-r m_{2}+\theta^{2}}{2 r R\left(\rho-2 r+\theta^{2}\right)\left(n_{1}\left(m_{2}-1\right)-1\right)}(1-2 R \Gamma)^{1-n_{1}},
$$

and, from (3.15), we have

$$
C_{2}=\frac{1-2 R \Gamma}{m_{2}}\left(\widetilde{x}-\frac{\Gamma}{r}\right)^{1-m_{2}}>0
$$

\section{Properties of the optimal policies}

In this section, we provide properties of the optimal policies without the assumption $\rho-2 r+\theta^{2}>0$ given in Koo et al. [8].

Lemma 1. If $\rho-2 r+\theta^{2}<0$, then $\rho-r m_{2}+\theta^{2}>0$.

Proof. Plugging $m=\left(\rho+\theta^{2}\right) / r$ into the function $f(\cdot)$ in $(3.5)$ implies

$$
\begin{aligned}
f\left(\frac{\rho+\theta^{2}}{r}\right) & =r\left(\frac{\rho+\theta^{2}}{r}\right)^{2}-\left(\rho+r+\frac{1}{2} \theta^{2}\right) \frac{\rho+\theta^{2}}{r}+\rho \\
& =\frac{\rho-2 r+\theta^{2}}{2 r} \theta^{2}<0 .
\end{aligned}
$$

Thus we have the following inequality: $m_{2}<\left(\rho+\theta^{2}\right) / r<m_{1}$, and consequently we see that $\rho-r m_{2}+\theta^{2}>0$.

Lemma 2. $X^{\prime}(c)$ is an increasing function for $\Gamma \leq c<1 /(2 R)$. 
Proof. We can easily see that $X^{\prime \prime}(c)=4 n_{1}\left(n_{1}-1\right) R^{2} D_{1}(1-2 R c)^{n_{1}-2}$. Obviously the sign of $X^{\prime \prime}(c)$ depends on the signs of $\left(n_{1}-1\right)$ and $D_{1}$. Noting that $g(1)=\rho-2 r+\theta^{2}$, we consider the cases when $g(1)>0$ and $g(1)<0$, respectively.

First, when $g(1)=\rho-2 r+\theta^{2}>0$, we find that $0<n_{1}<1$ and $\rho-m_{2} r+\theta^{2}>$ $\rho-2 r+\theta^{2}>0$ since $g(1)>0$ and $0<m_{2}<1$, respectively. Thus we have $D_{1}<0$. Second, when $g(1)=\rho-2 r+\theta^{2}<0$, we obtain $n_{1}>1$ and $\rho-r m_{2}+\theta^{2}>0$ by Lemma 1 . Thus we have $D_{1}>0$. Ultimately, we always obtain

$$
\frac{1-n_{1}}{\rho-2 r+\theta^{2}}>0 \quad \text { and } \quad\left(n_{1}-1\right) D_{1}>0 .
$$

Therefore we see that $X^{\prime \prime}(c)$ is always positive.

Lemma 3. The wealth function $X(c)$ is an increasing function for $\Gamma \leq c<$ $1 /(2 R)$.

Proof. By Lemma 2, we see that $X^{\prime}(c)$ is an increasing function for $\Gamma \leq c<$ $1 /(2 R)$. Now we want to show that $X^{\prime}(\Gamma)>0$. Note that

$$
\begin{aligned}
& X^{\prime}(\Gamma)=-2 n_{1} R D_{1}(1-2 R \Gamma)^{n_{1}-1}-\frac{1}{\rho-2 r+\theta^{2}} \\
& =-\frac{\left(\rho-r m_{2}+\theta^{2}\right) n_{1}}{r\left(\rho-2 r+\theta^{2}\right)\left(n_{1}\left(m_{2}-1\right)-1\right)}-\frac{1}{\rho-2 r+\theta^{2}} \\
& =-\frac{1}{\rho-2 r+\theta^{2}}\left\{\frac{\left(\rho-r m_{2}+\theta^{2}\right) n_{1} n_{2}}{r\left(n_{1}-n_{2}\right)}+1\right\} \\
& =-\frac{n_{1} n_{2}}{r\left(\rho-2 r+\theta^{2}\right)\left(n_{1}-n_{2}\right)}\left(\rho-r \frac{n_{2}+1}{n_{2}}+\theta^{2}-\frac{1}{2} \theta^{2}\left(n_{1}-n_{2}\right)\right) \\
& =-\frac{n_{1}}{r\left(\rho-2 r+\theta^{2}\right)\left(n_{1}-n_{2}\right)}\left(\rho n_{2}-r\left(n_{2}+1\right)+\theta^{2} n_{2}-\frac{1}{2} \theta^{2} n_{1} n_{2}+\frac{1}{2} \theta^{2} n_{2}^{2}\right) \\
& =-\frac{n_{1}}{r\left(\rho-2 r+\theta^{2}\right)\left(n_{1}-n_{2}\right)}\left\{\frac{1}{2} \theta^{2} n_{2}^{2}+\left(\rho-r+\frac{1}{2} \theta^{2}\right) n_{2}-r+\frac{1}{2} \theta^{2} n_{2}\left(1-n_{1}\right)\right\} \\
& =-\frac{\frac{1}{2} \theta^{2} n_{1} n_{2}\left(1-n_{1}\right)}{r\left(\rho-2 r+\theta^{2}\right)\left(n_{1}-n_{2}\right)}>0,
\end{aligned}
$$

where the third and the fourth equalities are obtained from the relationships between the roots (3.7) and Vieta's formula for the quadratic equation (3.6), the seventh equality from the fact that $n_{2}$ is one root of the quadratic equation (3.6), and the inequality from the first inequality in (4.1). Therefore $X^{\prime}(c)$ is positive since $X^{\prime}(c)$ is an increasing function for $\Gamma \leq c<1 /(2 R)$.

\section{Lemma 4.}

$$
\frac{1}{1-2 R \Gamma}\left[-\frac{1}{2 R n_{1}^{2} D_{1}\left(\rho-2 r+\theta^{2}\right)}\right]^{\frac{1}{n_{1}-1}}=\left[-\frac{\left(\rho-r m_{2}+\theta^{2}\right) n_{1}^{2} n_{2}}{r\left(n_{1}-n_{2}\right)}\right]^{\frac{1}{1-n_{1}}}<1
$$


Proof. If we substitute $D_{1}$ in (3.18) into the left-hand side of the above equation (4.2) and use the relationships in (3.7), we can obtain the equality. Let us consider

$$
\begin{aligned}
- & \frac{\left(\rho-r m_{2}+\theta^{2}\right) n_{1}^{2} n_{2}}{r\left(n_{1}-n_{2}\right)}=-\frac{n_{1}^{2} n_{2}}{r\left(n_{1}-n_{2}\right)}\left[\rho-r \frac{n_{2}+1}{n_{2}}+\theta^{2}-\frac{1}{2} \theta^{2}\left(n_{1}-n_{2}\right)\right]+n_{1} \\
& =-\frac{n_{1}^{2}}{r\left(n_{1}-n_{2}\right)}\left[\frac{1}{2} \theta^{2} n_{2}^{2}+\left(\rho-r+\frac{1}{2} \theta^{2}\right) n_{2}-r+\frac{1}{2} \theta^{2} n_{2}\left(1-n_{1}\right)\right]+n_{1} \\
& =n_{1}\left(1-n_{2}\right) /\left(n_{1}-n_{2}\right),
\end{aligned}
$$

where the first equality is obtained from the relationships in (3.7) and Vieta's formula for the quadratic equation (3.6), and the third equality from the fact that $n_{2}$ is one root of the quadratic equation (3.6) and Vieta's formula for the quadratic equation (3.6).

Now we consider two cases. When $0<n_{1}<1$, we see that

$$
n_{1}\left(1-n_{2}\right) /\left(n_{1}-n_{2}\right)<1
$$

and consequently we obtain

$$
\left[-\frac{\left(\rho-r m_{2}+\theta^{2}\right) n_{1}^{2} n_{2}}{r\left(n_{1}-n_{2}\right)}\right]^{\frac{1}{1-n_{1}}}=\left[\frac{n_{1}\left(1-n_{2}\right)}{n_{1}-n_{2}}\right]^{\frac{1}{1-n_{1}}}<1^{\frac{1}{1-n_{1}}}=1 .
$$

When $n_{1}>1$, we see that

$$
n_{1}\left(1-n_{2}\right) /\left(n_{1}-n_{2}\right)>1
$$

and consequently we obtain

$$
\left[-\frac{\left(\rho-r m_{2}+\theta^{2}\right) n_{1}^{2} n_{2}}{r\left(n_{1}-n_{2}\right)}\right]^{\frac{1}{1-n_{1}}}=\left[\frac{n_{1}\left(1-n_{2}\right)}{n_{1}-n_{2}}\right]^{\frac{1}{1-n_{1}}}<1^{\frac{1}{1-n_{1}}}=1 .
$$

Lemma 5. We have that $\widetilde{x}<\hat{x}<\bar{x}$, where

$$
\begin{aligned}
& \hat{\xi}:=\frac{1}{2 R}-\frac{1}{2 R}\left[-\frac{1}{2 R n_{1}^{2} D_{1}\left(\rho-2 r+\theta^{2}\right)}\right]^{\frac{1}{n_{1}-1}}, \\
& \hat{x}:=X(\hat{\xi})=D_{1}(1-2 R \hat{\xi})^{n_{1}}-\frac{1}{\rho-2 r+\theta^{2}} \hat{\xi}+\frac{\rho-r+\theta^{2}}{2 r R\left(\rho-2 r+\theta^{2}\right)} .
\end{aligned}
$$

Proof. It is enough to show that $\Gamma<\hat{\xi}<1 /(2 R)$ because the wealth function $X(c)$ is increasing for $\Gamma<c<1 /(2 R)$. From the definition of $\hat{\xi}$ in (4.3), we can easily check that $\hat{\xi}<1 /(2 R)$ since $-1 /\left(2 R n_{1}^{2} D_{1}\left(\rho-2 r+\theta^{2}\right)\right)>0$. Let us consider

$$
\hat{\xi}=\frac{1}{2 R}-\frac{1}{2 R}\left[-\frac{1}{2 R n_{1}^{2} D_{1}\left(\rho-2 r+\theta^{2}\right)}\right]^{\frac{1}{n_{1}-1}}>\frac{1}{2 R}-\frac{1-2 R \Gamma}{2 R}=\Gamma,
$$


where the inequality is obtained from (4.2) in Lemma 4.

The following theorem details the optimal consumption and portfolio policies and their properties without the assumption $\rho-2 r+\theta^{2}>0$ given in Koo et al. [8].

Theorem 2. The optimal consumption and portfolio policies $\left(c^{*}, \pi^{*}\right)$ are given by

$$
\begin{aligned}
& c_{t}^{*}=\left\{\begin{array}{l}
\Gamma, \quad \text { if } \Gamma / r<X_{t}<\widetilde{x}, \\
\xi_{t}, \quad \text { if } \widetilde{x} \leq X_{t}<\bar{x}, \\
1 / 2 R, \quad \text { if } X_{t} \geq \bar{x},
\end{array}\right. \\
& \pi_{t}^{*}=\left\{\begin{array}{l}
\frac{\theta}{\sigma\left(1-m_{2}\right)}\left(X_{t}-\frac{\Gamma}{r}\right), \quad \text { if } \Gamma / r<X_{t}<\widetilde{x}, \\
\frac{\theta}{\sigma}\left(-n_{1} D_{1}\left(1-2 R \xi_{t}\right)^{n_{1}}-\frac{1}{2 R\left(\rho-2 r+\theta^{2}\right)}\left(1-2 R \xi_{t}\right)\right), \quad \text { if } \widetilde{x} \leq X_{t}<\bar{x}, \\
0, \quad \text { if } X_{t} \geq \bar{x},
\end{array}\right.
\end{aligned}
$$

where $\xi_{t}$ is determined from the equation

$$
X_{t}=D_{1}\left(1-2 R \xi_{t}\right)^{n_{1}}-\frac{1}{\rho-2 r+\theta^{2}} \xi_{t}+\frac{\rho-2 r+\theta^{2}}{2 r R\left(\rho-2 r+\theta^{2}\right)} .
$$

Furthermore, we reaffirm the findings of Theorem 2.1 in Koo et al. [8], that $\pi_{t}^{*}$ increases for $\widetilde{x} \leq X_{t}<\hat{x}$, but $\pi_{t}^{*}$ decreases for $\hat{x} \leq X_{t}<\bar{x}$, without requiring the assumption $\rho-2 r+\theta^{2}>0$.

Proof. Using the FOCs (3.4) and (3.10) with the value function $V(\cdot)$ obtained in Theorem 1, we derive the optimal consumption and portfolio policies. For $\widetilde{x} \leq X_{t}<\bar{x}$, we define a function $h(\cdot)$ as follows:

$$
h\left(\xi_{t}\right):=\frac{d \pi_{t}^{*}}{d \xi_{t}}=\frac{\theta}{\sigma}\left\{2 R n_{1}^{2} D_{1}\left(1-2 R \xi_{t}\right)^{n_{1}-1}+\frac{1}{\rho-2 r+\theta^{2}}\right\} .
$$

Taking the derivative of $h(\cdot)$, we derive

$$
h^{\prime}\left(\xi_{t}\right)=-\frac{4 \theta R^{2} n_{1}^{2}\left(n_{1}-1\right) D_{1}}{\sigma}\left(1-2 R \xi_{t}\right)^{n_{1}-2} .
$$

By the second inequality in $(4.1), h^{\prime}(\cdot)$ is always negative, that is, $h(\cdot)$ is always decreasing. From the proof of Lemma 2 , for $\rho-2 r+\theta^{2}>0$, we have $0<n_{1}<1$ and $D_{1}<0$, and for $\rho-2 r+\theta^{2}<0$, we have $n_{1}>1$ and $D_{1}>0$, that is, $\left(\rho-2 r+\theta^{2}\right) D_{1}<0$. Thus $h(\cdot)=0$ has a unique solution $\hat{\xi}$ given in (4.3), and $\hat{x}:=X(\hat{\xi})$ is given in (4.4). Therefore we obtain, for $\widetilde{x} \leq X_{t}<\hat{x}, h(\cdot)>0$, and, for $\hat{x} \leq X_{t}<\bar{x}, h(\cdot)<0$. From Lemma $3, X(c)$ is an increasing function for $\Gamma \leq c<1 /(2 R)$, i.e., $d \xi_{t} / d X_{t}>0$. Thus, we obtain that for $\widetilde{x} \leq X_{t}<\hat{x}$, $d \pi_{t}^{*} / d X_{t}=d \pi_{t}^{*} / d \xi_{t} \cdot d \xi_{t} / d X_{t}=h\left(\xi_{t}\right) \cdot d \xi_{t} / d X_{t}>0$, and, for $\hat{x} \leq X_{t}<\bar{x}$, $d \pi_{t}^{*} / d X_{t}=d \pi_{t}^{*} / d \xi_{t} \cdot d \xi_{t} / d X_{t}=h\left(\xi_{t}\right) \cdot d \xi_{t} / d X_{t}<0$.

Theorem 3 [Verification Theorem]. $V(\cdot)$ in Theorem 1 satisfies the optimization problem (3.1). 
Proof. For any pair $(c, \pi) \in \mathcal{A}(x)$, we have

$$
\begin{aligned}
& \mathbb{E}\left[\int_{0}^{T} e^{-\rho t}\left(c_{t}-R c_{t}^{2}\right) d t\right] \leq \mathbb{E}\left[-\int_{0}^{T} e^{-\rho t}\left\{\left(r X_{t}+\pi_{t}(\mu-r)-c_{t}\right) V^{\prime}\left(X_{t}\right)\right.\right. \\
& \left.\left.\quad+\frac{1}{2} \sigma^{2} \pi_{t}^{2} V^{\prime \prime}\left(X_{t}\right)-\rho V\left(X_{t}\right)\right\} d t\right]=\mathbb{E}\left[-\int_{0}^{T} d\left(e^{-\rho t} V\left(X_{t}\right)\right)\right] \\
& \quad+\mathbb{E}\left[\int_{0}^{T} e^{-\rho t} \sigma \pi_{t} V^{\prime}\left(X_{t}\right) d B_{t}\right]=V(x)-e^{-\rho t} V\left(X_{T}\right)
\end{aligned}
$$

where the inequality is obtained from the Bellman equation (3.3), the first equality from Itô's formula to $e^{-\rho t} V\left(X_{t}\right)$, and the last equality from the fact that the second term of the left-hand side of the equality is a martingale.

Taking $T \rightarrow \infty$ and using the transversality condition (3.2), we obtain the following inequality:

$$
V(x) \geq \sup _{(c, \pi) \in \mathcal{A}(w)} \mathbb{E}\left[\int_{0}^{\infty} e^{-\rho t}\left(c_{t}-R c_{t}^{2}\right) d t\right]
$$

Similarly, for the pair $\left(c^{*}, \pi^{*}\right)$ of optimal strategies described in Theorem 2 , we have

$$
V(x)=\mathbb{E}\left[\int_{0}^{\infty} e^{-\rho t}\left\{c_{t}^{*}-R\left(c_{t}^{*}\right)^{2}\right\} d t\right] .
$$

Thus $V(\cdot)$ which is the solution to the Bellman equation (3.3) satisfies the optimization problem (3.1).

\section{Concluding remarks}

In this paper we consider a continuous-time optimal consumption and portfolio selection problem for an agent with quadratic utility and subsistence consumption constraints. We find the value function and optimal consumption and investment policies using the dynamic programming method. We are able to prove all our theorems and lemmas without an explicit assumption of $\rho-2 r+\theta^{2}>0$. As in Koo et. al [8], we find that an agent will consume at a subsistence consumption level for wealth below a certain threshold. Her consumption will increase monotonically with wealth for wealth levels above this threshold until it reaches a bliss level of consumption for which consumption does not increase with wealth. With regards to the agent's investment policy, the agent will invest a constant fraction of excess wealth into the risky asset for wealth levels that support the subsistence consumption. The agent's investment will increase at a decreasing rate for wealth levels above this threshold until it reaches its maximum at another threshold, and will decline with wealth for wealth levels above this threshold. At the wealth level which supports bliss consumption, the agent's investment in the risky asset will be zero.

\section{Acknowledgements}

The work of the first author (Yong Hyun Shin) was supported by the National Research Foundation of Korea (NRF) grant funded by the Korea govern- 
ment [Grant Nos. NRF-2013R1A1A2058027, NRF-2016R1A2B4008240] and the work of the corresponding author (Kum-Hwan Roh) was supported by the National Research Foundation of Korea (NRF) grant funded by the Korea government [Grant Nos.

NRF-2015R1C1A1A02037039, NRF-2017R1D1A3B03036548].

\section{References}

[1] S.T. Buccola. Portfolio selection under exponential and quadratic utility. Western Agricultural Economics Association, 7(1):43-52, 1982.

[2] J.C. Cox and C.-F. Huang. Optimal consumption and portfolio policies when asset prices follow a diffusion process. Journal of Economic Theory, 49(1):33-83, 1989. https://doi.org/10.1016/0022-0531(89)90067-7.

[3] P.H. Dybvig. Dusenberry's ratcheting of consumption: Optimal dynamic consumption and investment given intolerance for any decline in standard of living. The Review of Economic Studies, 62(2):287-313, 1995. https://doi.org/10.2307/2297806.

[4] N. Gong and T. Li. Role of index bonds in an optimal dynamic asset allocation model with real subsistence consumption. Applied Mathematics and Computation, 174(1):710-731, 2006. https://doi.org/10.1016/j.amc.2005.04.089.

[5] G. Hanoch and H. Levy. Efficient portfolio selection with quadratic and cubic utility. The Journal of Business, 43:181-189, 1970.

[6] C.-F. Huang and H. Pagès. Optimal consumption and portfolio policies with an infinite horizon: Existence and convergence. The Annals of Applied Probability, 2(1):36-64, 1992. https://doi.org/10.1214/aoap/1177005770.

[7] I. Karatzas, J.P. Lehoczky, S.P. Sethi and S.E. Shreve. Explicit solution of a general consumption/investment problem. Mathematics of Operations Research, 11(2):261-294, 1986. https://doi.org/10.1287/moor.11.2.261.

[8] J.L. Koo, S.R. Ahn, B.L. Koo, H.K. Koo and Y.H. Shin. Optimal consumption and portfolio selection with quadratic utility and a subsistence consumption constraint. Stochastic Analysis and Applications, 34(1):165-177, 2016. https://doi.org/10.1080/07362994.2015.1112748.

[9] P. Lakner and L.M. Nygren. Portfolio optimization with downside constraints. Mathematical Finance, 16(2):283-299, 2006. https://doi.org/10.1111/j.14679965.2006.00272.x.

[10] H.-S. Lee and Y.H. Shin. A dynamic programming approach to subsistence consumption constraints on optimal consumption and portfolio. Journal of Computational Analysis 83 Applications, 22(1):79-99, 2017.

[11] B.H. Lim, Y.H. Shin and U.J. Choi. Optimal investment, consumption and retirement choice problem with disutility and subsistence consumption constraints. Journal of Mathematical Analysis and Applications, 345(1):109-122, 2008. https://doi.org/10.1016/j.jmaa.2008.04.011.

[12] H.M. Markowitz. Foundations of portfolio theory. Journal of Finance, 46(2):469477, 1991. https://doi.org/10.1111/j.1540-6261.1991.tb02669.x.

[13] R.C. Merton. Lifetime portfolio selection under uncertainty: The continuoustime case. Review of Economics and Statistics, 51(3):247-257, 1969. https://doi.org/10.2307/1926560. 
[14] R.C. Merton. Optimum consumption and portfolio rules in a continuoustime model. Journal of Economic Theory, 3(4):373-413, 1971. https://doi.org/10.1016/0022-0531(71)90038-X.

[15] G. Shim and Y.H. Shin. Portfolio selection with subsistence consumption constraints and CARA utility. Mathematical Problems in Engineering, 2014, 2014. https://doi.org/10.1155/2014/153793.

[16] Y.H. Shin and B.H. Lim. Comparison of optimal portfolios with and without subsistence consumption constraints. Nonlinear Analysis: Theory, Methods $\&$ Applications, 74(1):50-58, 2011. https://doi.org/10.1016/j.na.2010.08.014.

[17] Y.H. Shin, B.H. Lim and U.J. Choi. Optimal consumption and portfolio selection problem with downside consumption constraints. Applied Mathematics and Computation, 188(2):1801-1811, 2007. https://doi.org/10.1016/j.amc.2006.11.053.

[18] H. Yuan and Y. Hu. Optimal consumption and portfolio policies with the consumption habit constraints and the terminal wealth downside constraints. Insurance: Mathematics and Economics, 45(3):405-409, 2009. https://doi.org/10.1016/j.insmatheco.2009.08.012. 